\title{
BIOMASSA DE MUDAS DE PEPINOS HÍBRIDOS CONDUZIDOS SOB AMBIENTES PROTEGIDOS $\left({ }^{1}\right)$
}

\author{
EDILSON COSTA $\left({ }^{2 *}\right)$; PAULO ADEMAR MARTINS LEAL $\left({ }^{3}\right)$; VIVIANE DO AMARAL GOMES $\left({ }^{2}\right)$; \\ DANIEL MACHADO $\left({ }^{2}\right)$; MURILLO CÉSAR DE SOUZA JARA $\left({ }^{2}\right)$
}

\begin{abstract}
RESUMO
O estudo de oleráceas no Estado de Mato Grosso do Sul, especialmente na região do Pantanal, é fundamental para o desenvolvimento do comércio local e sustentabilidade dos pequenos produtores que circunvizinham as áreas urbanas. Neste contexto, foi desenvolvido experimento com produção de mudas de pepino, estudando o acúmulo de biomassa aérea e radicular dos híbridos Aladdin F1, Nikkey, Safira e Nobre F1, em diferentes ambientes de cultivo e substratos. O experimento foi realizado em 2007, utilizando três ambientes de cultivo: (A1) estufa plástica; (A2) viveiro telado com sombrite® e (A3) viveiro telado com aluminet ${ }^{\circledR}$. Foram utilizadas três composições de substratos S1 (solo + fibra de coco); S2 (solo + pó-de-serra) e S3 (solo + composto orgânico). A resposta dos híbridos de pepinos em termos de biomassa seca das mudas dependeu do substrato e do ambiente de cultivo. O substrato "solo e fibra de coco" promoveu maior acúmulo de biomassa na estufa e no viveiro com tela de monofilamento. $\mathrm{O}$ substrato "solo e composto orgânico" proporcionou maior biomassa aérea no viveiro com aluminet ${ }^{\circ}$. O híbrido 'Safira' acumulou maior biomassa radicular nos telados e no substrato "solo e fibra de coco". O híbrido 'Nikkey' acumulou maior biomassa radicular no viveiro com aluminet ${ }^{\circledR}$ e no substrato "solo e fibra de coco", sem diferir de "solo e pó-de-serra". Os híbridos 'Aladdin F1' e 'Nobre F1' acumularam biomassa radicular similar nos ambientes; 'Aladdin F1' teve maior acúmulo com os substratos "solo e composto orgânico" e "solo e fibra de coco", e 'Nobre F1', maior acúmulo com "solo e fibra de coco", sem diferir de "solo e pó-de-serra".
\end{abstract}

Palavras-chave: ambiência, Cucumis sativus,substratos.

$\left.{ }^{1}\right)$ Recebido para publicação em $1 .^{\circ}$ de setembro de 2008 e aceito em 8 de outubro de 2009.

$\left({ }^{2}\right)$ Universidade Estadual de Mato Grosso do Sul - UEMS/Unidade de Aquidauana, Rodovia Aquidauana - Cera, Km 12, Caixa Postal 25, 79200-000 Aquidauana (MS). E-mail: mestrine@uems.br ${ }^{*}$ ) Autor correspondente.

$\left({ }^{3}\right)$ Universidade Estadual de Campinas, Faculdade de Engenharia Agrícola, FEAGRI/UNICAMP/CIPT - Av. Marechal Rondon, 501, Barão Geraldo, 13083-975 Campinas (SP). E-mail: pamleal@feagri.unicamp.br 


\title{
ABSTRACT \\ BIOMASS OF HYBRID CUCUMBER SEEDLINGS IN PROTECTED ENVIRONMENTS
}

\begin{abstract}
The study of vegetable productionn the State of Mato Grosso do Sul, particularly at the Pantanal region, is important for the development and sustainability of the local commercial and small farmers that live around cities. In this context experiment was developed with cucumber seedlings production, studying the accumulation of aerial and roots biomass of the Aladdin F1, Nikkey, Safira and Nobre F1 hybrids in different cultivation environments and substrates. The experiment was carried out in 2007 using three environments of cultivation: (A1) greenhouse; (2) nursery screen with sombrite ${ }^{\circledR}$ and (A3) nursery screen with aluminet ${ }^{\circledR}$. Three substrate compositions were used, S1 $(50 \%$ soil $+50 \%$ coconut fiber), S2 (50\% soil $+50 \%$ sawdust) and S3 (50\% soil $+50 \%$ organic compost). The responses of the cucumbers hybrids in terms of seedlings mass depended on the substrate and environment of cultivation. The substrate "soil and coconut fiber" increased biomass accumulation of the hybrids when used the greenhouse and nursery with monofilament screen. The substrate "soil and organic compost" showed greatest biomass in the nursery with aluminet ${ }^{\circledR}$. The 'Safira' hybrid accumulated more root biomass in screened environment and substrate "soil and coconut fiber". The 'Nikkey' hybrid accumulated more root biomass in the nursery with aluminet ${ }^{\circledR}$ and substrate "soil and coconut fiber", with no differences from "soil and sawdust" substrate. The 'Aladdin F1' and 'Nobre F1' hybrids accumulated similar root biomass in the environments, and the 'Aladdin F1' had higher accumulation with the substrates "soil and organic compost" and "soil and coconut fiber", while the 'Nobre F1' had higher accumulation in the "soil and coconut fiber" substrate with no differences from "soil and sawdust".
\end{abstract}

Key words: environment, Cucumis sativus, substrates.

A cadeia produtiva de hortaliças de qualidade começa com os cuidados que vão desde a formação das mudas até os tratamentos de pós-colheita. SEABRA JúnIOR et al. (2004) comentam que a base da horticultura moderna é a produção de mudas de alta qualidade, e mudas bem formadas dão origem a plantas com alto potencial produtivo.

Na propagação por mudas o substrato tem um importante papel na sua formação (NegreIros et al., 2003), pois, proporciona condições adequadas à germinação e ao sistema radicular (TeSSARIOLI Neto, 1994). Silva et al. (2001) comentam que os substratos devem ter as seguintes características: facilidade de transporte e disponibilidade, ausência de patógenos, riqueza de nutrientes, $\mathrm{pH}$ adequado, boa textura e estrutura.

Outro requisito importante na produção de mudas é a utilização de ambientes protegidos (SEGOVIA et al., 1997; SousA et al., 1997). Esses ambientes, geralmente, são cobertos com filme plástico de polietileno de baixa densidade, tela de sombreamento de monofilamento (sombrite ${ }^{\circledR}$ ) e tela de sombreamento aluminizada termo-refletora (aluminet ${ }^{\circledR}$ ) ou outros tipos de cobertura, que proporcionam alterações no microclima do ambiente (SENTELHAS et al., 1998); esses materiais interferem nas respostas das plantas aos processos fisiológicos.

Verificando a escassez de produção de hortaliças no Estado de Mato Grosso do Sul (BolETIM ANUAL, 2007) e a importância da fase de produção de mudas dentro da cadeia produtiva, este trabalho teve como objetivo a avaliação dos parâmetros de biomassa de mudas de pepineiro em função de ambientes de cultivo e substratos, em Aquidauana (MS).

O experimento foi instalado em Aquidauana (MS), localizada à altitude de $147 \mathrm{~m}$, longitude de $55,67^{\circ}$ e latitude de $-20,45^{\circ}$, região de interface entre o Cerrado e o Pantanal, clima classificado como Cwa e precipitação pluvial variando de 1200 a $1700 \mathrm{~mm}$ anuais.

Utilizaram-se quatro híbridos Aladdin F1 (H1), Nikkey (H2), Safira (H3) e Nobre F1 (H4). A semeadura ocorreu em 11/4/2007 em bandejas de 128 células $\left(34,6 \mathrm{~cm}^{3}\right)$ com dimensões padronizadas de $37,5 \mathrm{~cm}$ de largura, $67,5 \mathrm{~cm}$ de comprimento e $6,2 \mathrm{~cm}$ de altura, e a coleta dos dados em 4/5/2007.

Foram utilizados três ambientes protegidos com dimensões de 5,0 m de comprimento por 5,0 m de largura por $2,50 \mathrm{~m}$ de pé-direito: (A1) estufa plástica, tipo capela, coberta com filme plástico de polietileno de baixa densidade, $150 \mathrm{~mm}$ de espessura, fechada na lateral frontalmente com tela de sombreamento de ráfia, malha para $50 \%$ de sombra; (A2) viveiro telado, com fechamentos na cobertura, frontal e lateral com tela de sombreamento de monofilamento, malha para $50 \%$ de sombra; (A3) viveiro telado, com fechamentos na cobertura, frontal e lateral com tela de sombreamento aluminizada (aluminet ${ }^{\circledR}$, malha para $50 \%$ de sombra).

Utilizaram-se três composições de substratos S1 (solo + fibra de coco, na proporção volumétrica de $1: 1 \mathrm{v} / \mathrm{v}) ; \mathrm{S} 2$ (solo + pó-de-serra, na proporção volumétrica de 1:1 v/v) e S3 (solo + composto orgânico, na proporção volumétrica de 1:1 v/v). 
Na preparação dos substratos, o solo foi obtido da área da Unidade Universitária de Aquidauana; o composto orgânico da empresa Organoeste ${ }^{\circledR} / \mathrm{MS}$ (Tabela 1), com aproximadamente 1,5 anos de fabricação; o pó-de-serra de madeireiras da região, com idade aproximada de um ano e a fibra de coco da empresa Amafibra ${ }^{\circledR} / \mathrm{SP}$.

Aplicaram-se doses de $2,5 \mathrm{~kg}$ de superfosfato simples, $0,3 \mathrm{~kg}$ de cloreto de potássio e $1,5 \mathrm{~kg}$ de calcário dolomítico, com base em um volume de $1 \mathrm{~m}^{3}$ de substrato. Os substratos ficaram em descanso por
7 (sete) dias. Aos vinte e três dias após a semeadura (DAS) foram mensuradas as massas secas da parte aérea (MSPA) e do sistema radicular (MSSR), em gramas.

Para a avaliação dos experimentos utilizouse o delineamento inteiramente casualizado em parcelas sub-subdivididas (split-split plot), com cinco repetições. As parcelas principais foram os ambientes de cultivo (A), as subparcelas, as composições de substratos (S) e as sub-subparcelas, os híbridos $(\mathrm{H})$.

Tabela 1. Análise química do solo e do composto orgânico utilizados no experimento

\begin{tabular}{|c|c|c|c|c|c|c|c|c|c|c|}
\hline & $\mathrm{pH}$ & M.O. & $\mathrm{P}$ & $\mathrm{K}$ & $\mathrm{Ca}$ & $\mathrm{Mg}$ & $\mathrm{H}+\mathrm{Al}$ & SB & $\mathrm{T}$ & $\mathrm{V}$ \\
\hline & $\mathrm{CaCl}_{2}$ & $\%$ & $\mathrm{mg} \mathrm{dm}^{-3}$ & 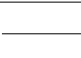 & 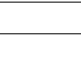 & $-\mathrm{cr}$ & $\mathrm{dm}^{-3}$ & & & $\%$ \\
\hline Solo & 5,4 & 1,4 & 10,5 & 0,35 & 0,9 & 0,8 & 3,3 & 17,4 & 20,7 & 38,3 \\
\hline Composto & 6,5 & 5,0 & 1924,1 & 2,62 & 15,7 & 0,7 & 2,7 & 19,0 & 21,7 & 87,7 \\
\hline
\end{tabular}

Fonte: Laboratório de análises do solo da agência estadual de defesa sanitária animal e vegetal de MS.

M.O. = matéria orgânica; $\mathrm{P}=$ fósforo; $\mathrm{K}=$ potássio; $\mathrm{Ca}=$ cálcio; $\mathrm{Mg}=$ magnésio; $\mathrm{H}+\mathrm{Al}=$ acidez potencial; $\mathrm{SB}=$ soma de bases; $\mathrm{T}=$ capacidade de troca de cátions; $\mathrm{V}=$ saturação por bases.

Os dados foram submetidos à análise de variância e as médias ao teste de Tukey, a 5\% de probabilidade. As análises foram realizadas pelo programa computacional EstAT (UNESP/FCAV, 1994).

Para a variável massa seca do sistema radicular (MSSR) houve interações entre ambientes, substratos e híbridos, no entanto, para a massa seca foliar (MSPA) houve interação, apenas, entre ambientes e substratos.

Verificou-se comportamento diferenciado dos substratos nos diversos ambientes. Não houve influência dos ambientes para as variáveis MSPA e MSSR do substrato S1 e MSPA do substrato S2. Para a MSSR do substrato S2 a estufa plástica (A1) foi o ambiente que promoveu menores massas, destacandose os telados (A2 e A3). No substrato S3 verifica-se que o ambiente A3 (tela termorrefletora) foi melhor que os demais, para as variáveis estudadas (Tabela 2).
PINTo et al. (2007) concluíram que o telado aluminizado, com $40 \%$ de sombra, promoveu melhor desenvolvimento em plantas de Alfazema-do-Brasil, que telado com níveis de sombra de $80 \%$, evidenciando que as espécies respondem de maneira distinta ao tipo de sombreamento.

O desenvolvimento das mudas foi afetado pelos ambientes e pelos substratos concordando com os comentários de CAÑIzares et al. (2002), que destacaram a utilização de substratos à base de terra ou vermiculita para a produção de mudas de pepino japonês. Os diferentes materiais utilizados em coberturas de ambientes protegidos proporcionaram alterações em seus microclimas (SENTELHAS et al., 1998), interferindo nas respostas das plantas aos processos fisiológicos, como fotossíntese e transpiração (ZANELLA et al., 2006).

Tabela 2. Massa seca da parte aérea (MSPA) e do sistema radicular (MSSR) de mudas de pepino aos 23 DAS para os diversos substratos (S) e ambientes (A) estudados

\begin{tabular}{|c|c|c|c|c|c|c|}
\hline & \multicolumn{3}{|c|}{ MSPA } & \multicolumn{3}{|c|}{ MSSR } \\
\hline & A1 & A2 & A3 & A1 & A2 & A3 \\
\hline & & & & $x_{2}$ & & 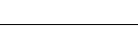 \\
\hline S1 & $0,077 \mathrm{Aa}$ & 0,089 Аa & 0,073 Ba & 0,027 Aa & 0,030 Aa & $0,030 \mathrm{Aa}$ \\
\hline S2 & $0,050 \mathrm{Ba}$ & 0,059 Ba & 0,056 Ca & $0,017 \mathrm{Bc}$ & $0,029 \mathrm{Aa}$ & $0,021 \mathrm{Bb}$ \\
\hline S3 & 0,041 Bc & $0,067 \mathrm{Bb}$ & 0,090 Аa & $0,019 \mathrm{Bc}$ & $0,023 \mathrm{Bb}$ & 0,031 Aa \\
\hline
\end{tabular}

* Médias seguidas de mesma letra maiúscula na coluna e minúscula na linha, não diferem entre si pelo teste de Tukey, a $5 \%$ de probabilidade. $\mathrm{S} 1$ = "solo + fibra de coco"; S2 = "solo + pó-de-serra"; S3 = "solo + composto orgânico"; A1 = estufa plástica; A2 = viveiro telado com sombrite ${ }^{\circledR} ; \mathrm{A} 3=$ viveiro telado com aluminet ${ }^{\circledR}$. 
Dentro do ambiente A1 o substrato S1 proporcionou os maiores valores. No ambiente A2 o substrato $\mathrm{S} 1$ continuou se destacando, visto que não difere do substrato S2 para a MSSR. No ambiente A3, destacou-se o substrato S3 para a MSPA e os substratos S3 e S1 para a MSSR (Tabela 2).

No ambiente A3 o substrato S3, que continha composto orgânico, promoveu desenvolvimento de mudas numericamente semelhantes ao promovido pelo substrato S1, que continha fibra de coco, evidenciando que pode ser uma alternativa de material a ser utilizado na produção de mudas de pepineiro na região de Aquidauana (Tabela 2).

Modolo e Tessarioli Neto (1999) verificaram, em mudas de quiabeiro produzidas em bandejas com células de maior volume, os melhores resultados com relação ao seu desenvolvimento para todos os parâmetros estudados. A adição de casca de arroz carbonizada no substrato comercial, na proporção 1:1, proporcionou menor desenvolvimento das mudas. A utilização de suplementação mineral ao substrato comercial favoreceu o desenvolvimento das mudas de quiabeiro.

O substrato "solo + pó-de-serra, na proporção volumétrica de 1:1 v/v" (S2) promoveu os menores acúmulos de biomassa no ambiente A3, provavelmente por demandar maior suprimento de nitrogênio no processo de estabilização, de acordo com o observado por Boff et al. (2005) em cebolas.

Outro efeito que pode ter ocorrido com o substrato pó-de-serra é o efeito alelopático das substâncias químicas que poderiam estar contidas nesse material (neolignanas), as quais, dependendo de suas concentrações, podem ter propiciado a inibição do crescimento e desenvolvimento das plantas, conforme destacado por BorGEs et al. (2007).
Em estufa plástica coberta com filme de polietileno em Botucatu (SP), SEABRA JÚNIOR et al. (2004), trabalhando com o substrato comercial Mercplant, cobrindo as sementes de pepino com palha de arroz carbonizada para evitar a evaporação excessiva de água das células da bandeja, constataram valores de massa seca da parte aérea (MSPA) superiores aos valores obtidos no presente experimento realizado em Aquidauana. Os pesquisadores avaliaram a idade das mudas e o volume dos recipientes, constatando aos 24 dias após a semeadura (DAS), na bandeja de 128 células, 0,3 g de massa seca (parte aérea + raiz), enquanto no presente trabalho, para o substrato S1 no ambiente A2, verificou-se 0,12 g aos 23 DAS (Tabela 2). Em bandeja com maior volume de substrato (72 células) os autores observaram valores de $0,5 \mathrm{~g}$.

Para a MSSR verifica-se que os híbridos não diferiram entre si no ambiente A1. No ambiente A2, o híbrido $\mathrm{H} 3$ não diferiu do híbrido $\mathrm{H} 1$, e propriciou maior valor que os híbridos $\mathrm{H} 2$ e H4. No ambiente A3 o híbrido H2, que não diferiu do híbrido H3, proporcionou maior valor de MSSR que os híbridos H1 e H4 (Tabela 3).

Pelos valores de MSSR, observa-se que no ambiente A2, respectivamente, os híbridos $\mathrm{H} 2$ e H1 sem diferirem de $\mathrm{H} 4$, e $\mathrm{H} 2$ e $\mathrm{H} 4$ sem diferirem de $\mathrm{H} 1$ tiveram os piores desenvolvimentos (Tabela 3 ). Galvani (2001) destaca que as temperaturas elevadas associadas a ventos constantes aumentam o estresse nas plantas diminuindo seu desenvolvimento, fato observado com maior intensidade no ambiente A1 na média das temperaturas máximas (Tabela 4 ).

Tabela 3. Massa seca do sistema radicular de mudas de pepino aos 23 DAS para os diversos híbridos (H) nos ambientes (A) e nos substratos (S) estudados

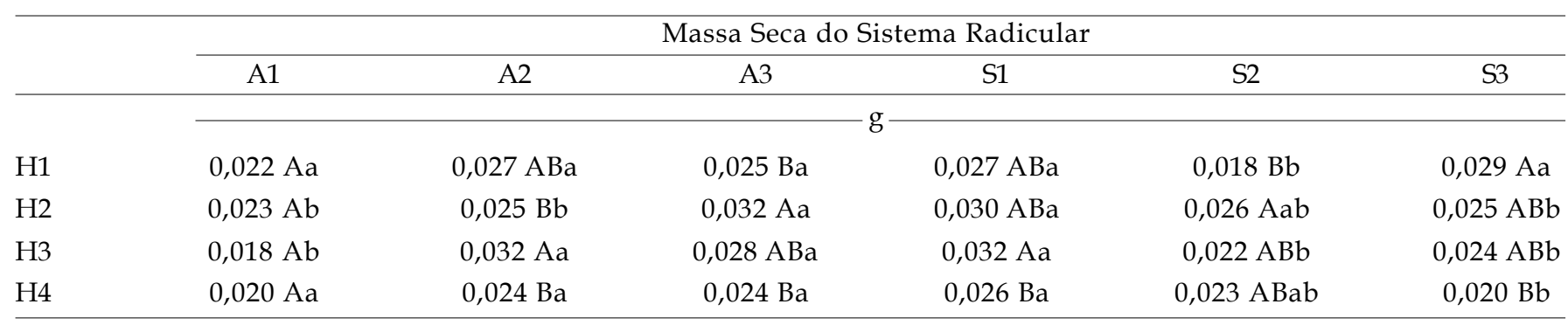

* Médias seguidas de mesma letra maiúscula na coluna e minúscula na linha, não diferem entre si pelo teste de Tukey, a $5 \%$ de probabilidade. $\mathrm{H} 1$ = Aladdin F1; H2 = Nikkey; H3 = Safira; H4 = Nobre F1; A1 = estufa plástica; $\mathrm{A} 2$ = viveiro telado com sombrite ${ }^{\circledR} ; \mathrm{A} 3=$ viveiro telado com Aluminet ${ }^{\circledR} ; \mathrm{S} 1$ = "solo + fibra de coco"; S2 = "solo + pó-de-serra"; S3 = "solo + composto orgânico". 
Tabela 4. Média das temperaturas máximas (Tmax) e mínimas (Tmin) nos ambientes de cultivo (A), no período experimental

\begin{tabular}{lcc}
\hline Ambientes & Tmax & Tmin \\
\cline { 2 - 3 } A1 & 39,1 & \\
A2 & 36,9 & 20,6 \\
A3 & 36,3 & 20,5 \\
\hline
\end{tabular}

$\mathrm{A} 1$ = estufa plástica; $\mathrm{A} 2$ = viveiro telado com sombrite ${ }^{\circledR} ; \mathrm{A} 3$ = viveiro telado com aluminet ${ }^{\circledR}$.

SMiderle et al. (2001), em Piracicaba/SP, combinando "Plantmax ${ }^{\circledR}+$ Solo + Areia" observaram massa seca da parte aérea (MSPA) e massa seca do sistema radicular (MSSR) de mudas de pepinos superiores às massas constantes do presente experimento. Aos 21 e 40 DAS obtiveram valores de MSPA, variando de 1,13-2,48 g e 2,09-5,64 g, e de MSSR variando de 0,62-1,58 g e 1,33-2,34 g respectivamente. Porém, os autores não comentam se o ambiente utilizado foi climatizado ou não.

Para a MSSR, verifica-se, para a maioria dos híbridos estudados, que o substrato S1 promoveu melhor desenvolvimento radicular que os demais substratos. Para o híbrido H1 esse substrato não diferiu do substrato S3, e para o híbrido $\mathrm{H} 2$ não diferiu do substrato S2 (Tabela 3).

A resposta dos híbridos de pepinos em termos de biomassa seca das mudas dependeu do substrato e do ambiente de cultivo. O substrato "solo e fibra de coco" promoveu maior acúmulo de biomassa na estufa e no viveiro com tela de monofilamento. O substrato "solo e composto orgânico" promoveu maior biomassa aérea no viveiro com aluminet ${ }^{\circledR}$. O híbrido 'Safira' acumulou maior biomassa radicular nos telados e no substrato "solo e fibra de coco". O híbrido 'Nikkey' acumulou maior biomassa radicular no viveiro com aluminet ${ }^{\circledR}$ e no substrato "solo e fibra de coco", sem diferir de "solo e pó-de-serra". Os híbridos 'Aladdin F1' e 'Nobre F1' acumularam biomassa radicular similar nos ambientes; 'Aladdin F1' teve maior acúmulo com os substratos "solo e composto orgânico" e "solo e fibra de coco", enquanto 'Nobre F1' teve maior acúmulo com "solo e fibra de coco", sem diferir de "solo e pó-de-serra".

\section{REFERÊNCIAS}

BOFF, P.; DEBARBA, J.F.; SILVA, E.; WERNER, H. Qualidade e sanidade de mudas de cebola em função da adição de composto termófilo. Horticultura Brasileira, v.23, p.875-880, 2005.
BOLETIM ANUAL 2007. CEASA/MS. Disponível em: www.ceasa.ms.gov.br. Acesso em 3 dez. 2008.

BORGES, F.C.; SANTOS, L.S.; CORREAA, M.J.C.; OLIVEIRA, M.N.; SOUZA FILHO, A.P.S. Potencial alelopático de duas neolignanas isoladas de folhas de virola surinamensis (myristicaceae). Planta Daninha, v.25, p.51-59, 2007.

CAÑIZARES, K.A.L.; COSTA, P.C.; GOTO, R.; VIEIRA, A.R.M. Desenvolvimento de mudas de pepino em diferentes substratos com e sem uso de solução nutritiva. Horticultura Brasileira, v.20, p.227-229, 2002.

GALVANI, E. Avaliação agrometeorológica do cultivo de pepino (Cucumis sativus L.) em ambientes protegido e a campo, em ciclos de outono-inverno e primaveraverão. Botucatu, 2001. 124p. Tese (doutorado)-FCA, UNESP.

MODOLO, V.A.; TESSARIOLI NETO, J. Desenvolvimento de mudas de quiabeiro [Abelmoschus esculentus (L.) Moench] em diferentes tipos de bandeja e substrato. Scientia agricola, v.56, p.377-381, 1999.

NEGREIROS, J.R.S.; ÁLVARES, V.S.; BRAGA, L.R.; BRUCKNER, C.H. Diferentes substratos na formação de mudas de maracujazeiro-amarelo. Revista Ceres, v.51, 243249, 2003.

PINTO, J.E.B.P.; CARDOSO, J.C.W.; CASTRO, E.M.; BERTOLUCCI, S.K.; MELO, L.A.; DOUSSEAU, S. Aspectos morfofisiológicos e conteúdo de óleo essencial de plantas de alfazema-do-Brasil em função de níveis de sombreamento. Horticultura Brasileira, v.25, p.210-214, 2007.

SEABRA JÚNIOR, S.; GADUM, J.; CARDOSO, A.I.I. Produção de pepino em função da idade das mudas produzidas em recipientes com diferentes volumes de substrato. Horticultura Brasileira, v.22, p.610-613, 2004.

SEGOVIA, J.F.O.; ANDRIOLO, J.L.; BURIOL, G.A.; SCHNEIDER, F.M. Comparação do crescimento e desenvolvimento da alface (Lactuca sativa L.) no interior e no exterior de estufas de polietileno em Santa Maria, RS. Ciência Rural, v.27, p.37-41, 1997.

SENTELHAS, P.C.; VILLA NOVA, N.A.; ANGELOCCI, L.R. Efeito de diferentes tipos de cobertura, em mini-estufas, na atenuação da radiação solar e da luminosidade. Revista Brasileira de Agrometeorologia, v.6, p.479-481, 1998.

SILVA, R.P.; PEIXOTO, J.R.; JUNQUEIRA, N.T.V. Influência de diversos substratos no desenvolvimento de muda de maracujazeiro-azedo (Passiflora edulis Sims f. flavicarpa Deg). Revista Brasileira de Fruticultura, v.23, p.377-381, 2001.

SMIDERLE, O.J.; SALIBE, A.B.; HAYASHI, A.H.; MINAMI, K. Produção de mudas de alface, pepino e pimentão em substratos combinando areia, solo e Plantmax ${ }^{\circledR}$. Horticultura Brasileira, v.19, p.253-257, 2001. 
SOUSA, J.A.; LEDO, F.J.S.; SILVA, M.R. Produção de mudas de hortaliças em recipientes. Embrapa Acre, 1997. 20p. (Circular Técnica 19)

TESSARIOLI NETO, J. Mudas olerícolas de alta qualidade. In: MINAMI, K; TESSARIOLI NETO, J.; PENTEADO, S.R.; SCARPARI, F.J. A produção de mudas hortícolas de alta qualidade. Piracicaba: Gráfica Universitária de Piracicaba, 1994. p.10-15.

UNIVERSIDADE ESTADUAL PAULISTA “JÚLIO DE MESQUITA FILHO". Departamento de Ciências Exatas. ESTAT. Versão 2.0. Jaboticabal: FCAV/UNESP, 1994.

ZANELLA, F.; SONCELA, R.; LIMA, A.L.S. Formação de mudas de maracujazeiro "amarelo" com níveis de sombreamento em Ji-Paraná/RO. Ciência e Agrotecnologia, v.30, p.880-884, 2006. 